\title{
Multifunctional Property Improvements by Combining Graphene and Conventional Fillers in Chlorosulfonated Polyethylene Rubber Composites
}

Bettina Strommer, Alexander Battig, Daniele Frasca, Dietmar Schulze, Christian Huth, Martin Böhning, Bernhard Schartel*

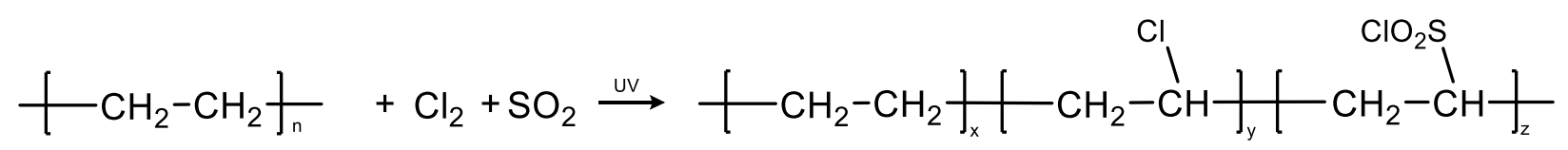
Scheme S1. Chemical reaction: chlorination and sulfochlorination of polyethylene to CSM.

Table S1. Composition of CSM composites in parts per hundred rubber (phr).

\begin{tabular}{lllllll}
\hline & CSM & CSM & CSM & CSM & CSM & CSM \\
& $20 / 0 / 0$ & $20 / 0 / 50$ & $5 / 3 / 0$ & $5 / 3 / 50$ & $20 / 3 / 47$ & $5 / 3 / 47$ \\
\hline CSM & 100 & 100 & 100 & 100 & 100 & 100 \\
CB & 20 & 20 & 5 & 5 & 20 & 5 \\
MLG & - & - & 3 & 3 & 3 & 3 \\
ATH & - & 50 & - & 50 & 47 & 47 \\
\hline
\end{tabular}

Table S2. Curing characteristics of CSM composites.

\begin{tabular}{lllllll}
\hline & CSM & CSM & CSM & CSM & CSM & CSM \\
& $20 / 0 / 0$ & $20 / 0 / 50$ & $5 / 3 / 0$ & $5 / 3 / 50$ & $20 / 3 / 47$ & $5 / 3 / 47$ \\
\hline $\mathrm{t}_{\mathrm{s} 1} / \min$ & $1.05 \pm 0.2$ & $0.63 \pm 0.1$ & $1.03 \pm 0.1$ & $0.67 \pm 0.0$ & $0.61 \pm 0.1$ & $0.70 \pm 0.1$ \\
$\mathrm{t}_{\mathrm{s} 2} / \mathrm{min}$ & $1.35 \pm 0.2$ & $0.75 \pm 0.1$ & $1.18 \pm 0.2$ & $0.86 \pm 0.1$ & $0.72 \pm 0.1$ & $0.81 \pm 0.1$ \\
$\mathrm{t}_{\mathrm{c} 90} / \mathrm{min}$ & $10.6 \pm 1.0$ & $6.32 \pm 0.8$ & $9.75 \pm 1.5$ & $6.38 \pm 0.8$ & $5.63 \pm 0.6$ & $6.45 \pm 1.0$ \\
$\mathrm{ML} / \mathrm{dNm}$ & $0.92 \pm 0.0$ & $1.72 \pm 0.1$ & $0.92 \pm 0.1$ & $1.86 \pm 0.1$ & $2.57 \pm 0.1$ & $1.84 \pm 0.1$ \\
$\mathrm{MH} / \mathrm{dNm}$ & $18.9 \pm 0.3$ & $29.5 \pm 0.1$ & $19.9 \pm 0.1$ & $29.0 \pm 0.5$ & $34.2 \pm 0.9$ & $28.2 \pm 0.5$ \\
$\Delta \mathrm{S} / \mathrm{dNm}$ & $17.9 \pm 0.2$ & $27.7 \pm 0.1$ & $19.0 \pm 0.1$ & $27.1 \pm 0.6$ & $31.6 \pm 0.9$ & $26.4 \pm 0.5$ \\
$v / 10^{-5} \mathrm{~mol} \mathrm{~g}^{-1}$ & 11.9 & 19.8 & 16.1 & 32.7 & 37.5 & 30.7 \\
\hline
\end{tabular}

$\mathrm{t}_{\mathrm{s} 1}$ and $\mathrm{t}_{\mathrm{s} 2}$ : scorch times, $\mathrm{t}_{\mathrm{c} 90}$ : optimum curing time, ML: minimum torque, $\mathrm{MH}$ : maximum torque, $\Delta \mathrm{S}$ : difference in torque $\mathrm{MH}-\mathrm{ML}, v$ : apparent crosslinking density after Flory-Rehner 
eq. 1. Flory-Rehner equation ${ }^{1}$.

$$
v\left(m o l g{ }^{-1}\right)=-\frac{\ln \left(1-v_{r}\right)+v_{r}+\chi v_{r}}{2 \rho_{r} V_{s}\left(\sqrt[3]{v_{r}}-\frac{v_{r}}{2}\right)}=\frac{1}{2 M_{c}}
$$

$v$...apparent crosslink density in mol $\mathrm{g}^{-1}$

$v_{r} \ldots$ volume fraction of the equilibrium swollen rubber

$\chi$...Flory-Huggins polymer-solvent interaction parameter (CSM/Toluene: 0.422$)^{2}$

$\rho_{r} \ldots$ density of the rubber in $\mathrm{g} \mathrm{cm}^{-3}$

$V_{s}$...molar volume of the solvent (toluene: $106.27 \mathrm{~cm}^{3} \mathrm{~mol}^{-1}$ )

$M_{c}$...average molecular rubber weight between the crosslinking points in $\mathrm{g} \mathrm{mol}^{-1}$

Table S3. Mechanical properties of CSM composites, results from tensile tests.

\begin{tabular}{|c|c|c|c|c|c|c|}
\hline & $\begin{array}{l}\text { CSM } \\
20 / 0 / 0\end{array}$ & $\begin{array}{l}\text { CSM } \\
20 / 0 / 50\end{array}$ & $\begin{array}{l}\text { CSM } \\
5 / 3 / 0\end{array}$ & $\begin{array}{l}\text { CSM } \\
5 / 3 / 50\end{array}$ & $\begin{array}{l}\text { CSM } \\
20 / 3 / 47\end{array}$ & $\begin{array}{l}\text { CSM } \\
5 / 3 / 47\end{array}$ \\
\hline $\begin{array}{l}\text { Tensile } \\
\text { strength / MPa }\end{array}$ & $19.0 \pm 1.4$ & $19.6 \pm 1.2$ & $15.1 \pm 0.8$ & $17.5 \pm 1.0$ & $16.8 \pm 1.5$ & $16.3 \pm 1.1$ \\
\hline $\begin{array}{l}\text { Stress at } 100 \% \\
\text { strain / } \mathrm{MPa}\end{array}$ & $2.6 \pm 0.1$ & $6.1 \pm 0.1$ & $3.9 \pm 0.1$ & $6.0 \pm 0.1$ & $8.6 \pm 0.1$ & $5.6 \pm 0.1$ \\
\hline $\begin{array}{l}\text { Elongation at } \\
\text { break / \% }\end{array}$ & $312 \pm 21$ & $317 \pm 19$ & $351 \pm 10$ & $354 \pm 14$ & $235 \pm 24$ & $360 \pm 13$ \\
\hline $\begin{array}{l}\text { Young's } \\
\text { modulus / } \mathrm{MPa}\end{array}$ & $4.43 \pm 0.2$ & $9.16 \pm 0.9$ & $6.59 \pm 0.1$ & $10.8 \pm 1.1$ & $15.8 \pm 0.2$ & $11.9 \pm 0.5$ \\
\hline
\end{tabular}

Table S4. Overview of the results of DMA measurements of CSM composites.

\begin{tabular}{lllllll}
\hline & CSM & CSM & CSM & CSM & CSM & CSM \\
& $20 / 0 / 0$ & $20 / 0 / 50$ & $5 / 3 / 0$ & $5 / 3 / 50$ & $20 / 3 / 47$ & $5 / 3 / 47$ \\
\hline $\begin{array}{l}\mathrm{E}^{6} \text { at }-40{ }^{\circ} \mathrm{C} \\
/ \mathrm{MPa}\end{array}$ & $1110 \pm 14$ & $1725 \pm 7$ & $1225 \pm 7$ & $1895 \pm 148$ & $2070 \pm 127$ & $1905 \pm 120$ \\
$\mathrm{E}^{6}$ at $25{ }^{\circ} \mathrm{C}$ & $2.28 \pm 0.1$ & $4.50 \pm 0.2$ & $3.35 \pm 0.2$ & $6.52 \pm 0.2$ & $9.57 \pm 0.3$ & $6.63 \pm 0.2$ \\
$/ \mathrm{MPa}$ & & & & & & \\
$\max \tan \delta /-$ & $1.6 \pm 0.1$ & $1.4 \pm 0.1$ & $1.4 \pm 0.1$ & $1.3 \pm 0.1$ & $1.1 \pm 0.1$ & $1.3 \pm 0.1$ \\
$\mathrm{~T}_{\mathrm{g}} /{ }^{\circ} \mathrm{C}$ & -8.6 & -8.1 & -9.1 & -8.5 & -8.6 & -8.6 \\
Hardness & $59.6 \pm 0.1$ & $73.4 \pm 0.2$ & $64.0 \pm 0.3$ & $74.2 \pm 0.1$ & $79.7 \pm 0.2$ & $73.9 \pm 0.4$ \\
$/$ Shore A & & & & & & \\
\hline
\end{tabular}




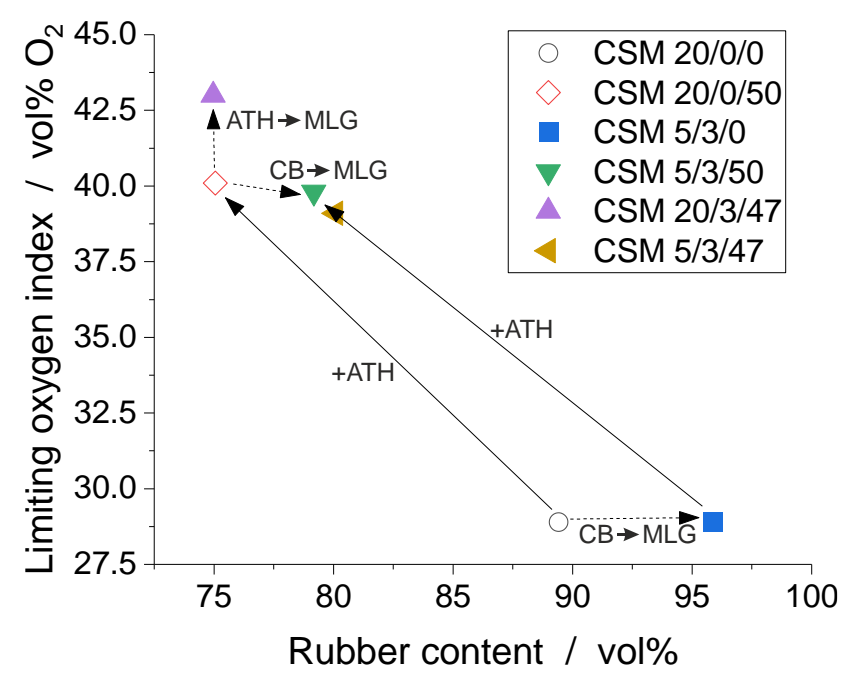

Figure S1. Limiting oxygen index vs. rubber content.

Table S5. Results of LOI and U1-94 measurements as the reaction to small flames.

\begin{tabular}{lllllll}
\hline & CSM & CSM & CSM & CSM & CSM & CSM \\
& $20 / 0 / 0$ & $20 / 0 / 50$ & $5 / 3 / 0$ & $5 / 3 / 50$ & $20 / 3 / 47$ & $5 / 3 / 47$ \\
\hline LOI / vol $\%$ & $28.9 \pm 0.1$ & $40.1 \pm 0.1$ & $28.9 \pm 0.1$ & $39.8 \pm 0.2$ & $43.0 \pm 0.2$ & $39.1 \pm 0.2$ \\
UL-94 & V-1 & V-0 & V-1 & V-0 & V-0 & V-0 \\
\hline
\end{tabular}

Table S6. Results from cone calorimeter measurements.

\begin{tabular}{lllllll}
\hline & CSM & CSM & CSM & CSM & CSM & CSM \\
& $20 / 0 / 0$ & $20 / 0 / 50$ & $5 / 3 / 0$ & $5 / 3 / 50$ & $20 / 3 / 47$ & $5 / 3 / 47$ \\
\hline $\mathrm{t}_{\mathrm{ig}} / \mathrm{s}$ & $47 \pm 4$ & $63 \pm 1$ & $47 \pm 4$ & $58 \pm 3$ & $66 \pm 1$ & $60 \pm 2$ \\
Residue / $\mathrm{wt} \%$ & $52 \pm 1$ & $57 \pm 3$ & $42 \pm 2$ & $65 \pm 1$ & $60 \pm 1$ & $63 \pm 3$ \\
$\mathrm{PHRR} / \mathrm{kW} \mathrm{m}^{-2}$ & $221 \pm 4$ & $153 \pm 2$ & $217 \pm 5$ & $131 \pm 2$ & $121 \pm 1$ & $134 \pm 2$ \\
$\mathrm{THE} / \mathrm{MJ} \mathrm{m}^{-2}$ & $30.0 \pm 3.5$ & $28.7 \pm 2.1$ & $36.9 \pm 2.3$ & $23.7 \pm 1.5$ & $25.0 \pm 1.3$ & $23.9 \pm 2.3$ \\
$\mathrm{EHC} / \mathrm{kW} \mathrm{m}^{-2}$ & $16.5 \pm 0.3$ & $15.3 \pm 0.1$ & $17.5 \pm 0.4$ & $15.4 \pm 1.1$ & $14.2 \pm 0.5$ & $14.9 \pm 0.3$ \\
$\mathrm{TSP} / \mathrm{m}^{-2}$ & $22.0 \pm 1.3$ & $11.7 \pm 0.9$ & $25.8 \pm 1.0$ & $9.2 \pm 0.1$ & $10.0 \pm 0.4$ & $9.5 \pm 0.3$ \\
$\mathrm{MARHE} / \mathrm{kW} \mathrm{m}^{-2}$ & $130 \pm 1$ & $75 \pm 1$ & $134 \pm 7$ & $70 \pm 2$ & $63 \pm 4$ & $68 \pm 3$ \\
\hline
\end{tabular}

tig: time to ignition; PHRR: peak heat release rate; THE: total heat evolved (=total heat release at flame out); EHC: effective heat of combustion (=total heat evolved / total mass loss); TSP: total smoke production; MARHE: maximum average rate of heat emission 
1. Flory, P. J.; Jr., J. R. Statistical Mechanics of Cross-Linked Polymer Networks II. Swelling. J. Chem. Phys. 1943, 11, 521-526.

2. Marković, G.; Jovanović, V.; Samaržija-Jovanović, S.; Marinović-Cincović, M.; Budinski-Simendić, J. Curing and Mechanical Properties of Chlorosulphonated Polyethylene Rubber Blends. Chem. Ind. Chem. Eng. Q. 2011, 17 (3), 315-321. 